\title{
Knowledge representation and intelligent fault diagnosis technology for power grid faults
}

\author{
Xiang $\mathrm{Li}^{1,}{ }^{1}$, Xin $\mathrm{Min}^{2}$, Zeng Yang ${ }^{1}$, Hongyu $\mathrm{He}^{1}$, and Jinfeng $\mathrm{Li}^{1}$ \\ ${ }^{1}$ Power Dispatching and Controlling Centre, Guangzhou Power Supply Co., Ltd., Guangzhou 510620, China \\ ${ }^{2}$ School of Electrical Engineering, South China University of technology, Guangzhou 510640, China
}

\begin{abstract}
Aiming at that the automatic fault diagnosis method is difficult to locate the fault causes under uncertain circumstances which include malfunctions of the equipment and wrong alarm messages, a knowledge model was proposed to describe the relationship, status and operation of the equipment. And based on the model, the action logic between equipment after accidents is expressed in the form of rules combined with predicate logic. The corresponding interpretation and checking results of the relative alarm messages are given by reasoning the accident chain under different fault hypothesis. And the optimal judgment result is obtained through the calculation of prior probability. The validity of the method is verified by a practical fault case.
\end{abstract}

\section{Introduction}

When the grid fails, the alarm messages would be uploaded to the dispatch centre through SCADA (Supervisory Control and Data Acquisition). The dispatchers judge the type of the fault based on the information, and then find the accident handling plan. However, the number of the messages is large, especially when the fault involved multiple substations. Therefore, some automatic fault diagnosis methods were proposed to achieve rapid screening of the alarm messages and determination of the fault causes which combine computer technology and artificial intelligence technology

The automatic fault diagnosis methods mainly include rule-based expert system [1-2], analytic model [3-4], Bayesian networks [5-6], Petri nets [7-8] and artificial neural networks [9-10]. The traditional expert system method expresses the relationship between protection and faulty equipment as production rules, and finds the cause of the fault by reasoning. This method is clear and interpretable. But the descriptive ability of the rules is finite and it usually gets combinatoric explosion under complex situation. And it is also difficult to carry out in the case of false alarms information and equipment malfunctions. Therefore, expert system method often cooperates with other methods such as information theory, which builds a channel model to describe the uncertainty in the information transfer process; The analytical method converts the fault facts and diagnostic rules into a mathematical model. And search the hypothesis that matches the fault feature best by solving the optimization problem. This method has a strict mathematical logic foundation and copes with the uncertain information in the fault well. However, the dimension of the model is usually too high and the relevant weights in the model are most based on subjective setting, so the efficiency and accuracy of the diagnosis cannot be guaranteed; Based on the artificial neural network method, the relevant protection and switching action information are taken as input, and the fault cause is taken as the output. The fault samples are used to train the diagnostic neural network, which avoids the explicit expression of the fault diagnosis knowledge. It has good tolerance for the reported error alarm information, but the interpretation of the result is poor and the training effect is difficult to guarantee under large-scale power grid. Petri net technology represents the topological relationship between devices and the logical action relationship between protection, circuit breakers and faulty devices in the form of directed graphs. It initializes the state of the petri network according to the fault information firstly and then the network state would be updated through continuous reasoning. Finally, the diagnosis result could be got under the steady state. The reasoning speed is fast, but it is less versatile and remodelling is required under different grid structures.

In summary, the current automatic diagnosis technology has two difficulties: firstly, the descriptive ability and maintainability of the knowledge model reflecting the fault judgment logic are difficult to combine. Most methods only consider the relationship between protection and primary equipment. The operation of the automatic device for power recovery after voltage loss is not taken into account. This makes it difficult to explain the complete process of accident evolution and affects the accuracy of judgment. Secondly, the information source of the accident judgment often has false or unreported message, and the

\footnotetext{
* Corresponding author: 1ix@guangzhou.csg.cn
} 
related equipment also has the possibility of refusal and maloperation. These uncertain factors need to be considered in the automatic diagnosis of the accident. In view of the above two points, this paper firstly defined a fault diagnosis knowledge model that can fully describe the fault state, and then it achieves automatic diagnosis by reasoning the fault chain under the uncertainty based on the model. Finally, it found the most possible interpretation of the fault by calculating the probability of the chain.

\section{The knowledge representation model of fault diagnosis}

When a grid fault occurs, according to detecting the change in electrical quantity, the relevant primary and secondary equipment trigger the action response and achieve the isolation and recovery of the fault under a certain action logic. These data constitute the source information of the fault diagnosis. The fault diagnosis is to realize the mapping from the source information to the faulty device. So this requires some way to express the knowledge involved in the fault diagnosis, including the topology relationship between the devices, the relationship between the faulty equipment and the protection, the relationship between the protection and the action switch, the relationship between the protection and protection as well as the logic of the relevant automatic devices in the power grid. The entire accident evolution process can be fully described and the fault diagnosis can be realized only by expressing these logical relationships adequately and accurately. In this paper, based on the thought of the object-oriented, the abstract classification of object categories is performed according to the operational and functional characteristics of the grid equipment object in the situation of fault firstly. And then the ontology representation of fault diagnosis knowledge is formed by the logical association and attribute relationship between these object classes. Finally, through the instantiation of the grid object, the knowledge map of the fault diagnosis is gradually generated from the top to the bottom.

\subsection{The hierarchical division of concepts in grid}

The electrical quantity of the primary equipment changes suddenly when the power grid fails. At the same time, the secondary equipment detects the change of the operating parameters and triggers the action, controls the corresponding switch displacement to achieve the isolation of the faulty device and the recovery of the pressure-loss equipment. Based on the functional and operational characteristics of different device objects in the process, the relevant grid equipment is divided into categories in this paper, and the specific classification result are shown in figure 1.

As shown in figure 1, according to the different functions in the power grid, the equipment is divided into primary and secondary device, in which the primary device is classified into breaking device and operating device. Breaking device is the bearer for realizing the change of the operation mode of the power grid, and it is also the operation object of the fault treatment measures. According to the breaking capacity, it is divided into circuit breaker and disconnector here. Operating device is the main body of function implementation in the grid operation, but it does not have the ability to change the operating state. The operating parameters of these equipment are the characteristics of the grid operating conditions. They are the protection objects of the secondary equipment, which are mainly classified into bus, main transformer and line. In the secondary device, only the automatic device, protection and reclosure class are considered here according to the implemented functions. The automatic device is a control device for restoring the power supply of the voltage loss device after the fault, including bus automatic device and the line automatic device. Protection is a control device that isolates faulty equipment when a fault occurs. According to the different protection triggering principles, it is divided into distance protection, differential protection, overcurrent protection, etc. In addition, in order to cope with a large number of transient faults in the power grid, the lines often have reclosures. In order to be able to describe the relevant state, the reclosure class is defined here.

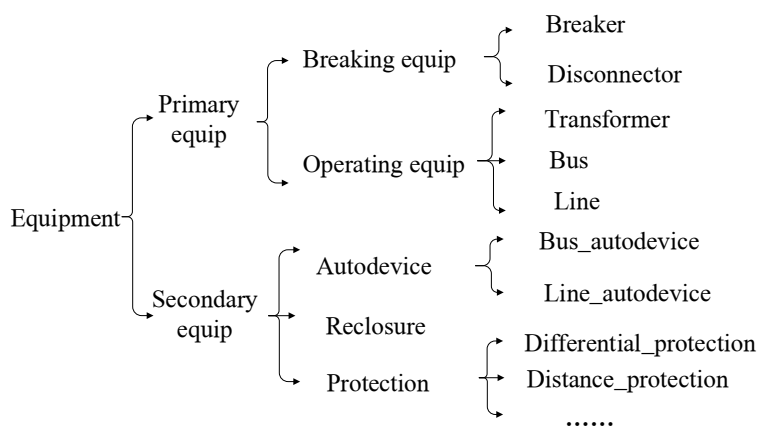

Fig. 1. Classification of electric network equipment.

\subsection{The definitions of device properties and relationships}

A large amount of operational data is generated in the real-time operation of the grid equipment. The information which the equipment attribute consisting of numerical values or strings reflects the operating status of the grid. On the other hand, there is a complex and close relationship between the grid equipment. After the fault occurs, the state change of the initial faulty equipment will trigger the action and change the attributes of all levels of equipment along the logical chain among the equipment, and finally change into the post-accident state. In order to express related information, based on the device concept categories classification as mentioned above, a constraint definition is defined on the attributes of each object concept and the relationship between the objects. And the representation format of the relationship between objects is defined as <object, attribute, attribute value, time $>$. Considering the time constraint relationship of the action logic between devices, a time label is added for each 
attribute, which the time of the device action attribute is the action time, and the time label of the device status attribute is the measured time. The specific device attributes are defined as the following table 1.

Table 1. Object attribute definition

\begin{tabular}{|c|c|c|}
\hline Attribute name & Object class & Attribute value \\
\hline Switch_state & Breaking equip & Closed/Open \\
\hline $\begin{array}{l}\text { Operating equip } \\
\text { state }\end{array}$ & Operating equip & $\begin{array}{c}\text { Run/Maintenanc } \\
\mathrm{e}\end{array}$ \\
\hline $\begin{array}{c}\text { Secondaryequip } \\
\text { state }\end{array}$ & Secondary equip & Off/On \\
\hline Functional_state & Equipment & Fault/Normal \\
\hline Current value & Primary equip & number \\
\hline Voltage value & Operating equip & number \\
\hline Operation_1 & Breaking equip & $\begin{array}{l}\text { Trip/Closing/ } \\
\text { No-action }\end{array}$ \\
\hline Operation_2 & Secondary equip & $\begin{array}{l}\text { Action / } \\
\text { No-action }\end{array}$ \\
\hline
\end{tabular}

The representation format of the relationship between objects is defined as <object, relationship, object>. The specific definition is as shown in figure 2 , in which each type of object relationship is represented by a pointing line segment, from the relational subject to the relational object. For example, the object relation original source from automatic device (auto device class) points to function equip (operating equip class). The relationship subject that represents this relationship is constrained to the automatic device, and the relationship object is constrained to the operating device. The specific meaning is the relationship between the automatic device and its primary disconnected battery.

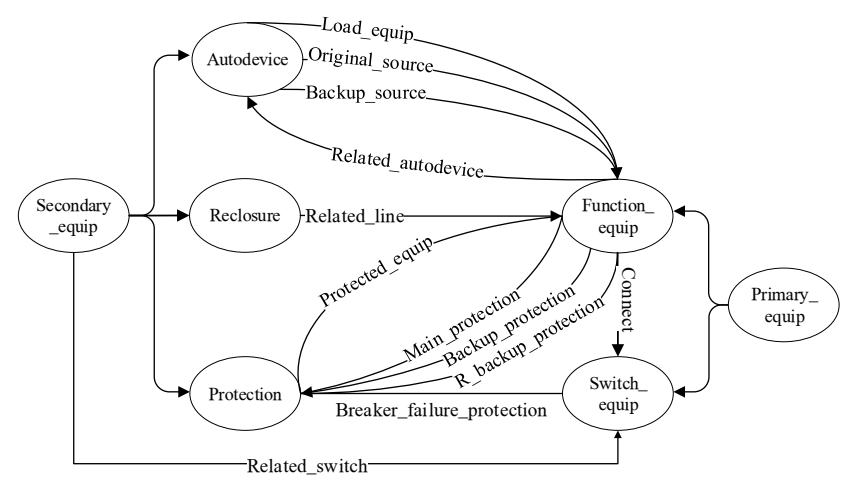

Fig. 2. Classification of electric network equipment.
In summary, the model definitions of equipment types, equipment relationships and equipment attributes in the power grid are given. Based on these, the grid state and equipment actions can be accurately described in the fault situation, which constitute the basic knowledge models of grid accident diagnosis.

\section{Fault chain reasoning considering uncertainty}

When the fault occurred in grid, the relative data would be uploaded to the dispatch centre through Supervisory Control and Data Acquisition (SCADA) system. These data reflect the operation of primary and secondary equipment which is the basis of the fault diagnosis. However, there are usually malfunction of relative equipment and transmission of fake data after original fault happen. The uncertainty makes the fault diagnosis difficult. Based on the fault diagnosis knowledge model above and action logic of the equipment in the fault, this paper achieves the diagnosis under uncertainty by the comparison between the fault data and the fault chain which is deduce from original fault assumption.

\subsection{The basic principle of reasoning}

Combined with the knowledge model in section 2, the action logic of the relative equipment is shown as figure 3. The grid state after fault is deduced considering the functional state (fault or normal) of each relative equipment. In order to improve the efficiency of diagnosis, the action logic of equipment within double faults is mainly considered and triple or more equipment faults in the actual power grid is not considered in most cases which rarely occurs and would increase the logic complexity and uncertainty. The red field in figure 3 is the equipment state assumption, and the blue field is the device action response.

And according to the logic graph, the reasoning rules are expressed in form of predicate logic which is shown in table 2. 


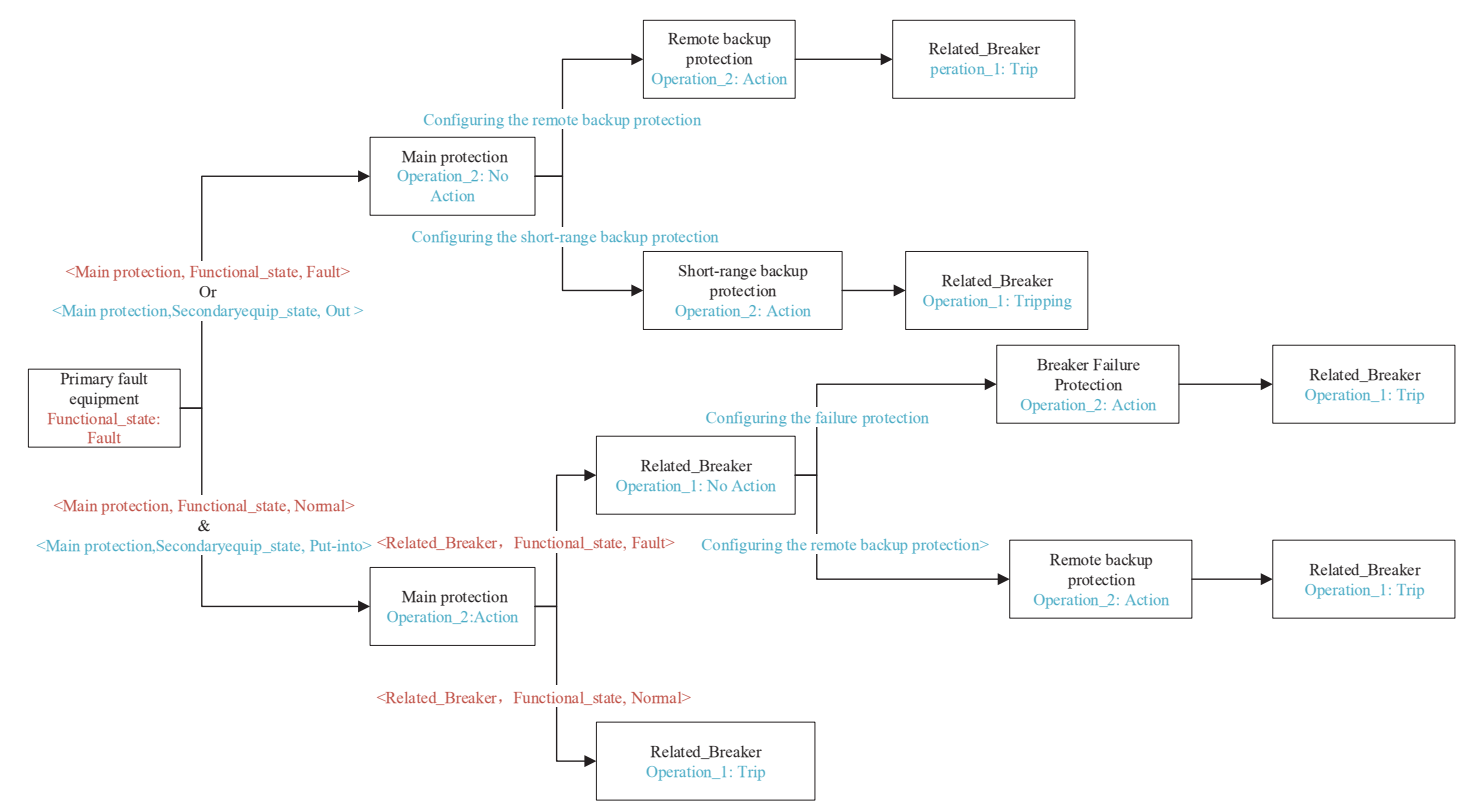

Figure 3. The action logic of relative protection and breakers after primary equipment fault

Table 2. The example of the rule expression

\begin{tabular}{|c|c|c|}
\hline Sequence & Rules condition & $\begin{array}{c}\text { Rules } \\
\text { conclusion }\end{array}$ \\
\hline 1 & $\begin{array}{l}\exists \mathrm{x}, \mathrm{y} .(\text { Primary_equip }(\mathrm{x}) \wedge \text { Functional_state }(\mathrm{x})= \\
\text { Fault } \wedge \text { Main_protection }(\mathrm{x}, \mathrm{y}) \wedge \text { Secondary } \\
\text { equip_state }(\mathrm{y})=\mathrm{On} \wedge \text { Functional_state }(\mathrm{y})= \\
\text { Normal })\end{array}$ & $\begin{array}{l}\text { Operation_2(y) } \\
=\text { Action }\end{array}$ \\
\hline 2 & $\begin{array}{l}\exists \mathrm{x}, \mathrm{y} .(\operatorname{Protection}(\mathrm{x}) \wedge \text { Operation_2(x)= Action } \\
\wedge \text { Breaker }(\mathrm{y}) \wedge \text { Related_breaker }(\mathrm{x}, \mathrm{y}) \wedge \\
\text { Functional_state }(\mathrm{y})=\text { Normal })\end{array}$ & $\begin{array}{l}\text { Operation_1(y) } \\
\text { = Trip }\end{array}$ \\
\hline 3 & $\begin{array}{l}\exists x, y, \text { z. }(\text { Primary_equip }(x) \wedge \text { Functional_state } \\
(x)=\text { Fault } \wedge \text { Main_protection } \quad(x, y) \wedge \\
\text { Functional_state }(y)=\text { Fault } \wedge \text { Short-range backup } \\
\text { protection }(x, z))\end{array}$ & $\begin{array}{l}\text { Operation_2(y) } \\
=\text { No-Action } \\
\text { Operation_2(z) } \\
\quad=\text { Action }\end{array}$ \\
\hline 4 & $\begin{array}{l}\exists \mathrm{x}, \mathrm{y}, \mathrm{z} .(\text { Protection }(\mathrm{x}) \wedge \text { Operation_2 }(\mathrm{x})=\text { Action } \\
\wedge \text { Breaker }(\mathrm{y}) \wedge \text { Related_breaker }(\mathrm{x}, \quad \mathrm{y}) \wedge \\
\text { Functional_state }(\mathrm{y})=\text { Fault } \wedge \text { Breaker } \\
\text { Protection }(\mathrm{y}, \mathrm{z}))\end{array}$ & $\begin{array}{l}\text { Operation_1(y) } \\
=\text { No-Action } \wedge \\
\text { Operation_2(z) } \\
\quad=\text { Action }\end{array}$ \\
\hline
\end{tabular}

After fault happened, the operation and state of the equipment in fault domain were got by analysing the data in SCADA. And then combined with the relations between the equipment which are defined in section 2 , the fact base is generated. The original fault assumption set are constituted by the primary equipment in voltage loss zone. And based on the fault development logic, the fault chain reasoning is carried out step by step from original fault assumption. The reasoning results of each step are compared with the fact database to judge the validity of process of reasoning and the truth of the data. The comparison rules are as follows:

If the actions or state attributes of two devices in the fact base are logically sequential, it is confirmed that relevant information is reported correctly and the latter device has normal functional attributes; For example, if a protective action is obtained by analysing teleportation message and the corresponding switch trip occurs, and the time of both actions meets the constraint, it is confirmed that both messages are reported correctly and the switch function is normal.

1) if the state or action attribute information of a certain device is obtained by analysing the telephony information, but there is no other device action or state information logically associated with it in the fact base, the information is considered to be a false alarm and the device has not actually acted.

2 ) if the action or state attributes of the device obtained by accident chain reasoning are consistent with 
the fact base, the relevant hypothesis is confirmed to be correct;

3 ) if there is no action or state information corresponding to the inference result in the fact database, but there is action or state information of upper and lower level devices logically related to the fact database, the relevant attribute information of the device is considered not reported and the relevant hypothesis is correct, otherwise, the inference is considered wrong;

4) for the complete accident chain obtained by inference, if the device action information in the fact base is not included in the accident chain and the device action information cannot be checked, it is considered that the device information is misreported; if other device information is checked with each other, it is considered that the relevant device acted wrong under the accident chain;

\subsection{Choice of inference result}

If multiple accident chains satisfying logical constraints are obtained through accident diagnostic reasoning, but the probability of occurrence of faults is often quite different. Here, the most probable cause of fault is selected as the diagnosis result by calculating the probability of occurrence of faults in each accident chain. According to the above analysis, the fault categories are statistically as follows. Protection fault; C. Standby selfswitching fault; D. Reclosing fault; It is assumed that the occurrence probability of the above mentioned faults is $P_{a}, P_{b}, P_{c}$ and $P_{d}$ respectively, and the number of the corresponding fault devices in the accident chain is $n_{a}$,

$n_{b} 、 n_{c}$ and $n_{d}$ respectively, then the calculation formula of the occurrence probability of the accident chain is as follows:

$$
P_{\text {chain }}=\prod_{i=a}^{d} P_{i}^{n_{i}}
$$

Since all values of Pi are relatively small, in order to facilitate calculation and comparison, logarithm of both sides of equation (1) can be obtained as follows:

$$
\begin{aligned}
& \ln P_{\text {chain }}=\sum_{i=a}^{d} n_{i} \ln P_{i} \\
& E_{\text {chain }}=-\ln P_{\text {chain }}
\end{aligned}
$$

Here, the accident chain with the minimum $E_{\text {chain }}$ value is selected as the output result of fault diagnosis.

\section{Case verification}

In order to prove the validity of the above diagnostic principle, a $110 \mathrm{kV}$ regional grid is taken as an example to illustrate the fault diagnosis process. As shown in Figure 4, breaker 123 of line JPY is equipped with distance protection, zero-sequence protection and automatic reclosing. The main transformers of Sanzhi Substation and Nanpu Substation are equipped with differential protection, gas protection and backup protection. And the $10 \mathrm{kV}$ bus of each substation has automatic bus transfer.

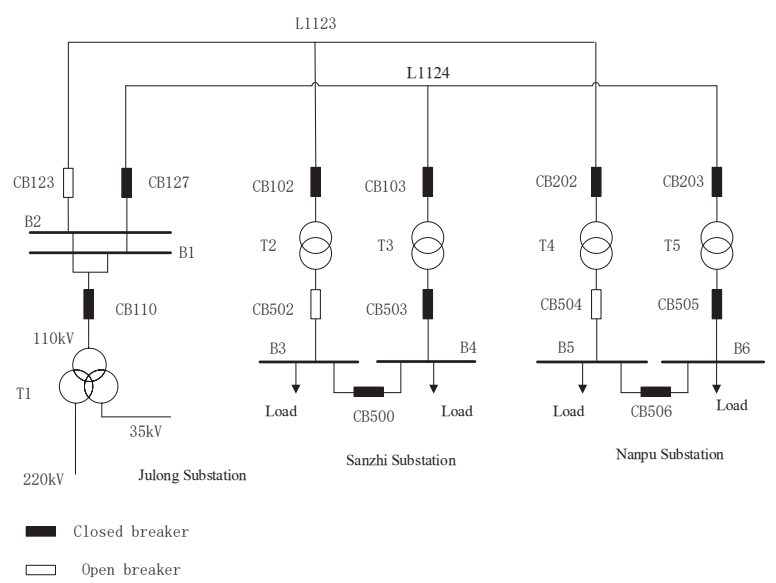

Figure 4. Structural Chart of Fault Power Network

After the fault occurs, the SCADA system receives

\begin{tabular}{|c|c|c|}
\hline Substation & Equipment & Operation \\
\hline Sanzhi & $\begin{array}{l}\text { differential protection of } \\
\mathrm{T} 2\end{array}$ & Action \\
\hline Sanzhi & Breaker CB502 & Trip \\
\hline Sanzhi & $\begin{array}{l}\text { automatic bus transfer } \\
\text { equipment of } \mathrm{B} 3 \text { and } \mathrm{B} 4\end{array}$ & Action \\
\hline Sanzhi & Breaker CB500 & Closing \\
\hline Julong & $\begin{array}{c}\text { Zero-sequence II } \\
\text { protection of L1123 }\end{array}$ & Action \\
\hline Julong & Breaker CB123 & Trip \\
\hline Julong & Reclosure of CB123 & Action \\
\hline Julong & Breaker CB123 & Closing \\
\hline Julong & Breaker CB123 & Trip \\
\hline Nanpu & $\begin{array}{l}\text { automatic bus transfer } \\
\text { equipment of B5 and B6 }\end{array}$ & Action \\
\hline Nanpu & Breaker CB504 & Trip \\
\hline Nanpu & Breaker CB506 & Closing \\
\hline Nanpu & $\begin{array}{c}\text { Zero-sequence } \\
\text { overcurrent protection of } \\
\text { T5 }\end{array}$ & Action \\
\hline
\end{tabular}
the fault alarm messages which is shown in table 3 :

Table 3. Alarm message of the fault

According to the outrage area after the fault, it is found that the fault primary equipment include line1123, transformer T2 in Sanzhi substation and transformer T4 in Nanpu substation. The initial fault hypothesis set is composed of equipment. Then the fault chain is reasoned separately. The results of the reasoning are shown as table 4:

Table 4. Results of Fault Chain Reasoning

\begin{tabular}{|c|lcc|c|}
\hline sequence & \multicolumn{3}{|c|}{ fault chain } & $\begin{array}{c}\text { faulty } \\
\text { equipment }\end{array}$ \\
\hline 1 & $\begin{array}{l}\text { Transformer T2 fault } \\
\text { differential protection of }\end{array}$ & T2 \\
\hline
\end{tabular}




\begin{tabular}{|l|l|}
\hline & \begin{tabular}{l} 
action $\rightarrow$ CB102 refused to \\
move CB502 trip $\rightarrow$ remote \\
backup protection of T2 (zero \\
sequence II protection of L1123) \\
action $\rightarrow$ CB123 trip $\rightarrow$ CB123 \\
unsuccessful reclosing, L1123 \\
outrage $\rightarrow$ B3 B5 outrage $\rightarrow$ \\
Automatic bus transfer equipment \\
action $\rightarrow$ CB504 trip, CB500、 \\
CB506 close $\rightarrow$ B3、 B5 recovery \\
power supply \\
\hline L1123 fault $\rightarrow$ main protection of \\
L1123 refused to act $\rightarrow$ backup \\
protection of L1123 act zero \\
sequence II protection) $\rightarrow$ CB123 \\
trip $\rightarrow$ CB123 unsuccessful \\
reclosing , L1123 outrage $\rightarrow$ \\
B3、B5 outrage $\rightarrow$ Automatic bus \\
transfer equipment action $\rightarrow$ \\
CB504、CB502 trip, CB500、 \\
CB506 close $\rightarrow$ B3、 B5 recovery \\
power supply
\end{tabular} \mid L1123 \\
\hline
\end{tabular}

As shown in the table above, there are two possible fault chains obtained by diagnostic reasoning. For the chain 1, it does not cover the protection information in Nanpu and there is no other alarm message associated with the action of the protection. So, the protection information is identified as wrong alarm message. For the accident chain 2, except for the wrong information in chain 1, the alarm message about differential protection of T2 was not covered in the accident chain either, but it was verified to be correctly reported according to the action of $\mathrm{CB} 502$, and $\mathrm{CB} 102$ was identified as refusing to act because of its closed state.

After comparing the fault equipment in the two accident chains, it can be found that the fault equipment in chain 1 is included in the fault equipment set of chain 2. According to the above formula for calculating the probability of fault, it can be inferred that the probability of occurrence of chain 1 must be higher than that of chain 2. Therefore, the final output result is that Sanzhi \#2 main transformer is faulty, the main protection of the transformer action and Breaker102 refused to open. The fault is removed by the remote backup protection (zerosequence II protection of line JPY). The information about the action of Nanpu \#1 main transformer zerosequence overcurrent protection is a fake alarm message. It can be seen that the fault diagnosis result is consistent with the manual judgment.

\section{Conclusion}

Through the summary of the development process of grid faults, this paper establishes a knowledge representation model for fault diagnosis. A knowledge representation method based on first-order predicate logic is provided by defining the equipment type, equipment attribute and equipment relationship which is used to describe fault characteristics and inference logic in fault diagnosis. And On the basis, summarize the logical relationship of equipment actions after the fault, as a rule, perform forward reasoning of the accident chain and check the message information of the faulty equipment, and determine the false alarm, unreported information, malfunction, and refusal equipment under the fault condition. Not only the accident diagnosis under uncertainty is realized, but also the evolution process of the fault is well reflected. The corresponding explanation of the equipment action in the fault range is given. Finally, the effectiveness of the diagnostic method is verified by the actual grid fault case.

\section{References}

1 Park Y M, Kim G W and Sohn J M 1997. A logic based expert system (LBES)for fault diagnosis of power system . IEEE Transaction on Power Systems. 12 pp 363-369.

2 Fukui C and Kawakami J 1986. An expert system for fault section estimation using information from protective relays and circuit breakers . IEEE Transactions on Power Delivery.1 pp 83-90.

3 Wenxin Guo, Fushuan Wen and Gerard Ledwich 2010. An Analytic Model for Fault Diagnosis in Power Systems Considering Malfunctions of Protective Relays and Circuit Breakers. IEEE Transactions on Power Delivery.25 pp 1393-1401.

4 Y. Zhang, C. Y. Chung, F. Wen and J. Zhong 2016 Analytic Model for Fault Diagnosis in Power Systems Utilizing Redundancy and Temporal Information of Alarm Messages, IEEE Transactions on Power Systems.31, pp 4877-4886.

5 Yongli, Z., H. Limin and L. Jinling 2006 Bayesian Networks-Based Approach for Power Systems Fault Diagnosis. IEEE Transactions on Power Delivery, 21 pp 634-639.

6 Cai, B., L. Huang and M. Xie 2017 Bayesian Networks in Fault Diagnosis. IEEE Transactions on Industrial Informatics. 13 pp 2227-2240.

7 W. xiaoli, C. guangju, X. yue and G. zhaoxin 2007 Proc. Int. Conf. on Electronic Measurement and Instruments, Fault Detection and Diagnosis Based on Time Petri Net,

8 L. Gong, R. Ma, H. Yang and Y. He 2015 Proc. Int. Conf. on Electric Utility Deregulation and Restructuring and Power Technologies, The substation fault diagnosis method based on the time constraint probability Petri net,

9 Thukaram D, Khincha P and Vijaynarasimha H P 2005 Artificial neural network and support vector machine approach for locating faults in radial distribution systems $[\mathrm{J}]$. IEEE Transactions on Power Delivery 20 pp 710-721.

10 Xiaoming Han and Yan Chen 2011 Proc. Int. Conf. on Electronic and Mechanical Engineering and Information Technology (EMEIT), GrC and ANN based fault diagnosis method of distribution network. 